\title{
ON MODULI SUBSETS OF CENTRAL EXTENSIONS OF RATIONAL H-SPACES
}

\author{
Takahito NAITO
}

(Received 28 February 2011 and revised 25 May 2011)

\begin{abstract}
We investigate the moduli sets of central extensions of $\mathrm{H}$-spaces enjoying inversivity, power associativity and Moufang properties. By considering rational $\mathrm{H}-$ extensions, it turns out that there is no relationship between the first and the second properties in general.
\end{abstract}

\section{Introduction}

We assume that a space has the homotopy type of a path connected CW-complex with a nondegenerate base point $*$ and that all maps are based maps.

An $H$-space is a space $X$ endowed with a map $\mu: X \times X \rightarrow X$, called a multiplication, such that both the restrictions $\left.\mu\right|_{X \times *}$ and $\left.\mu\right|_{* \times X}$ are homotopic to the identity map of $X$. The multiplication naturally induces a binary operation on the homotopy set $[Y, X]$ for any space $Y$. In [4], James proved that $[Y, X]$ is an algebraic loop; that is, it has a two-sided unit element and, for any elements $x$ and $y \in[Y, X]$, the equations $x a=y$ and $b x=y$ have unique solutions $a, b \in[Y, X]$.

Loop theoretic properties of $\mathrm{H}$-spaces have been considered by several authors; for example, Curjel [3] and Norman [7]. In [1], Arkowitz and Lupton considered H-space structures with inversive, power associative and Moufang properties. They consider whether there exists an H-space structure which does not satisfy the properties. Thanks to the general theory of algebraic loops, we see that the Moufang property implies inversivity and power associativity. However, it is expected that there is no relationship between the latter two properties in general.

In [5], Kachi introduced central extensions of $\mathrm{H}$-spaces which are called central H-extensions; see Definition 2.7. Roughly speaking, for a given homotopy associative and homotopy commutative $\mathrm{H}$-space $X_{1}$ and an $\mathrm{H}$-space $X_{2}$, a central $\mathrm{H}$-extension of $X_{1}$ by $X_{2}$ is defined to be the product $X_{1} \times X_{2}$ with a twisted multiplication. He also gave a classification theorem for the extensions. In fact, a quotient set of an appropriate homotopy set classifies the equivalence classes of central H-extensions; see Theorem 2.14. Such a quotient set is called the moduli set of H-extensions. Moreover, we refer to the subset of the moduli set corresponding to the set of the equivalence classes of $\mathrm{H}$-extensions enjoying a property $P$ via the bijection in the classifying theorem as the moduli subset of H-extensions associated with the property $P$.

2010 Mathematics Subject Classification: Primary 55P45; Secondary 55P62.

Keywords: H-space; algebraic loop; rational homotopy theory.

(C) 2012 Faculty of Mathematics, Kyushu University 
The objective of this paper is to investigate the moduli subsets of central extensions of $\mathrm{H}$-spaces associated with inversive, power associative or Moufang properties. If a given $\mathrm{H}$-space is $\mathbb{Q}$-local, then the moduli set of its central H-extensions is endowed with a vector space structure over $\mathbb{Q}$. It turns out that the moduli subset mentioned above inherits the vector space structure. This fact enables us to compare such moduli subsets as a vector space, and thus our main theorem (Theorem 4.7) deduces the following result.

ASSERTION 1.1. Let $S_{\mathrm{inv}}, S_{\mathrm{p} . \mathrm{a}}$ and $S_{\mathrm{Mo}}$ be the moduli subsets of central H-extensions of the Eilenberg-MacLane space $K(\mathbb{Q}, n)$ by $K(\mathbb{Q}, m)$ associated with inversive, power associative and Moufang properties, respectively. Here, the Eilenberg-MacLane space may be considered as a based loop space; that is, a homotopy associative and commutative H-space.

(1) If $m$ is even, $n=k m$ and $k \geq 4$ is even, then $S_{\mathrm{Mo}}$ is the proper subset of $S_{\mathrm{p} . \mathrm{a}}$ and $S_{\mathrm{p} . \mathrm{a}}$ is the proper subset of $S_{\mathrm{inv}}$ :

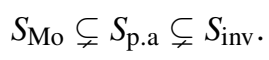

(2) If $m$ is even, $n=k m$ and $k \geq 5$ is odd, then $S_{\mathrm{Mo}}$ is the proper subset of $S_{\mathrm{p} . \mathrm{a}} \cap S_{\mathrm{inv}}$. Moreover, $S_{\mathrm{p} . \mathrm{a}} \cap S_{\mathrm{inv}}$ is a proper subset of $S_{\mathrm{p} . \mathrm{a}}$ and $S_{\mathrm{inv}}$ :

$$
S_{\text {Mo }} \subsetneq S_{\text {p.a }} \cap S_{\text {inv }} \subsetneq S_{\text {p.a }} \text { and } \quad S_{\text {p.a }} \cap S_{\text {inv }} \subsetneq S_{\text {inv }} .
$$

(3) Otherwise, $S_{\text {inv }}=S_{\text {p.a }}=S_{\text {Mo }}$.

Kachi [5] proved that the space $K(\mathbb{Z}, 2 n) \times S^{n}(n=1,3,7)$ admits infinitely many essentially different multiplications. As a corollary of Assertion 1.1, we have the following result.

COROLlARY 1.2. The product space $K(\mathbb{Z}, 2 n) \times S^{n}(n=1,3,7)$ has infinitely many essentially different multiplications which satisfy the Moufang property.

The organization of this paper is as follows. In Section 2, we will recall several fundamental definitions and facts on $\mathrm{H}$-spaces and algebraic loops. The classification theorem of central H-extensions are also described. In Section 3, we will present necessary and sufficient conditions for central H-extensions to be inversive, power associative and Moufang. The conditions allows us to describe the moduli set of extensions enjoying each of the properties in terms of a homotopy set of maps. In Section 4, we will deal with rational $\mathrm{H}$-spaces and the dimensions of the moduli sets mentioned above. Moreover, several examples are presented. Assertion 1.1 and Corollary 1.2 are proved at the end of Section 4.

\section{Preliminaries}

We begin by recalling the definition of an algebraic loop.

Definition 2.1. An algebraic loop $(Q, \cdot)$ is a set $Q$ with a map

$$
\cdot: Q \times Q \rightarrow Q ;(x, y) \longmapsto x y
$$

such that the equations $a x=b$ and $y a=b$ have unique solutions $x, y \in Q$ for all $a, b \in Q$. Moreover, there exists an element $e \in Q$ such that $x e=x=e x$ for all $x \in Q$. The element $e$ is called a unit. 
Algebraic loops with particular multiplications are considered.

Definition 2.2. An algebraic loop $(Q, \cdot)$ is called

(1) inversive if, for any element $x$ of $Q$, there exists a unique element $x^{-1}$ of $Q$ such that the equalities $x^{-1} x=e=x x^{-1}$ hold;

(2) power associative if $x(x x)=(x x) x$ for any $x \in Q$;

(3) Moufang if $(x(y z)) x=(x y)(z x)$ for any $x, y, z \in Q$; and

(4) flexible if $(x y) x=x(y x)$ for any $x, y \in Q$.

In Section 4, we will focus on the flexible property to examine the Moufang property.

LEMMA 2.3. [2, Lemma 2A] If an algebraic loop $Q$ is Moufang, then it is inversive.

Thus we have the following implication between properties for multiplication described in Definition 2.2:

$$
\text { power associative } \Longleftarrow \text { flexible } \Longleftarrow \text { Moufang } \Longrightarrow \text { inversive }
$$

We bring the loop theoretic notion to the realm of H-spaces.

Definition 2.4. Let $(X, \mu)$ be an H-space. A left inverse $l: X \rightarrow X$ and a right inverse $r: X \rightarrow X$ of an H-space $(X, \mu)$ are maps such that $\mu(l \times i d) \Delta \simeq * \simeq \mu(i d \times r) \Delta$.

We see that an $\mathrm{H}$-space has a left inverse and a right inverse unique up to homotopy [4].

Definition 2.5. An $\mathrm{H}$-space $(X, \mu)$ is called

(1) inversive if a left inverse and a right inverse of $(X, \mu)$ are homotopic;

(2) power associative if $\mu(\mu \times i d)(\Delta \times i d) \Delta \simeq \mu(i d \times \mu)(\Delta \times i d) \Delta$;

(3) Moufang if $\mu(\mu \times \mu) \theta \simeq \mu(\mu \times i d)(i d \times \mu \times i d) \theta$, where $\theta: X^{3} \rightarrow X^{4}$ is defined by $\theta(x, y, z)=(x, y, z, x)$; and

(4) flexible if $\mu(\mu \times i d)(i d \times t)(\Delta \times i d) \simeq \mu(i d \times \mu)(i d \times t)(\Delta \times i d)$, where $t: X^{2} \rightarrow$ $X^{2}$ is defined by $t(x, y)=(y, x)$.

The following proposition characterizes the above conditions on $\mathrm{H}$-spaces by homotopy sets.

Proposition 2.6. [1] Let $(X, \mu)$ be an H-space. Then $(X, \mu)$ is inversive (respectively, power associative, Moufang and flexible) if and only if the homotopy set $[Y, X]$ is inversive (respectively, power associative, Moufang and flexible) for any space $Y$.

Here we recall the definition of the central extension of an H-space.

Definition 2.7. [5] Let $\left(X_{1}, \mu_{1}\right)$ be a homotopy associative and homotopy commutative $\mathrm{H}$-space and let $\left(X_{2}, \mu_{2}\right)$ be an H-space. An H-space $(X, \mu)$ is a central H-extension of $\left(X_{1}, \mu_{1}\right)$ by $\left(X_{2}, \mu_{2}\right)$ if there exists a sequence of H-maps

$$
\left(X_{1}, \mu_{1}\right) \stackrel{f_{1}}{\rightarrow}(X, \mu) \stackrel{f_{2}}{\rightarrow}\left(X_{2}, \mu_{2}\right)
$$

such that the sequence

$$
e \longrightarrow\left[Y, X_{1}\right] \stackrel{f_{1 *}}{\longrightarrow}[Y, X] \stackrel{f_{2 *}}{\longrightarrow}\left[Y, X_{2}\right] \longrightarrow e
$$


is exact as algebraic loops for any space $Y$ and the image of $f_{1 *}$ is contained in the center of $[Y, X]$. Here $f_{i *}$ is the algebraic loop homomorphism induced by $f_{i}$ and the center of an algebraic loop $Q$ is the following subset of $Q[9$, p. 83]:

$$
\{x \in Q \mid r x=x r,(r s) x=r(s x),(x r) s=x(r s) \text { for any } r, s \in Q\} .
$$

In order to describe the classification theorem for central $\mathrm{H}$-extensions presented by Kachi, we recall the definition of H-deviations. Let $(X, \mu)$ be an $\mathrm{H}$-space and let $Y$ be a space. For any $[f],[g] \in[Y, X]$, let $D(f, g)$ be a unique element of $[Y, X]$ such that $D(f, g)[g]=[f]$.

Definition 2.8. [11, Definition 1.4.1] Let $(X, \mu)$ and $\left(Y, \mu^{\prime}\right)$ be H-spaces, and let $f: X \rightarrow Y$ be a map. An H-deviation of the map $f$ is an element

$$
H D(f) \in[X \wedge X, Y]
$$

such that $q^{*}(H D(f))=D\left(f \mu, \mu^{\prime}(f \times f)\right)$, where $q: X \times X \rightarrow X \wedge X$ is the quotient map.

The existence of H-deviations is ensured by the following lemma.

Lemma 2.9. [11, Lemma 1.3.5] Let $(X, \mu)$ be an $H$-space. Then, for any spaces $Y$ and $Z$,

$$
e \longrightarrow[Y \wedge Z, X] \stackrel{q^{*}}{\rightarrow}[Y \times Z, X] \stackrel{i^{*}}{\rightarrow}[Y \vee Z, X] \longrightarrow e
$$

is the short exact sequence as algebraic loops, where $i: Y \vee Z \rightarrow Y \times Z$ is the inclusion and $q: Y \times Z \rightarrow Y \wedge Z$ is the quotient map.

Since $i^{*} D\left(f \mu, \mu^{\prime}(f \times f)\right)=D\left(f \mu i, \mu^{\prime}(f \times f) i\right)=e$, there exists an element $H D(f) \in[X \wedge X, Y]$ such that $q^{*}(H D(f))=D\left(f \mu, \mu^{\prime}(f \times f)\right)$. If $f \simeq f^{\prime}: X \rightarrow Y$, then $H D(f)=H D\left(f^{\prime}\right)$. Therefore, the H-deviation map

$$
H D:[X, Y] \rightarrow[X \wedge X, Y]
$$

is defined by sending a class $[f]$ to the class $H D(f)$.

Lemma 2.10. Let $(X, \mu)$ and $\left(Y, \mu^{\prime}\right)$ be H-spaces and let $f: X \rightarrow Y$ be a map such that $[f \mu],\left[f p_{1}\right]$ and $\left[f p_{2}\right]$ are in the center of $[X \times X, Y]$, where $p_{1}$ and $p_{2}$ are the projections of $X \times X$ on the first and second factors respectively. Then, for any map $g: X \rightarrow Y$, $H D(f \cdot g)=H D(f) H D(g)$.

Remark 2.11. In [5, Lemma 2.3], Kachi claimed Lemma 2.10 with different hypotheses. However, it is necessary to modify the hypotheses and the proof of [5, Lemma 2.3], and hence we modify the assertion of Lemma 2.3 in [5] as in Lemma 2.10. We note that there is no effect on the arguments in paper [5], especially, on the argument of [5, p. 255], even if we replace Lemma 2.3 in [5] with Lemma 2.10.

Proof of Lemma 2.10. By the definition of the H-deviation, the equality $H D(f \cdot g)=$ $H(f) H(g)$ holds if and only if

$$
D\left((f \cdot g) \mu, \mu^{\prime}(f \cdot g \times f \cdot g)\right)=D\left(f \mu, \mu^{\prime}(f \times f)\right) D\left(g \mu, \mu^{\prime}(g \times g)\right)
$$


for any $f, g: X \rightarrow Y$. Since $\left[f p_{1}\right]$ and $\left[f p_{2}\right]$ are in the center, the equations $\left[\mu^{\prime}(f \times f)\right]=$ $\left[f p_{1}\right] \cdot\left[f p_{2}\right]$ and $\left[\mu^{\prime}(g \times g)\right]=\left[g p_{1}\right] \cdot\left[g p_{2}\right]$ show that

$$
\begin{aligned}
{\left[\mu^{\prime}(f \times f) \cdot \mu^{\prime}(g \times g)\right] } & =\left(\left[f p_{1}\right]\left[f p_{2}\right]\right)\left(\left[g p_{1}\right]\left[g p_{2}\right]\right) \\
& =\left(\left[f p_{1}\right]\left[g p_{1}\right]\right)\left(\left[f p_{2}\right]\left[g p_{2}\right]\right)=\left[\mu^{\prime}(f \cdot g \times f \cdot g)\right]
\end{aligned}
$$

in $[X \times X, Y]$. It means that

$$
D\left((f \cdot g) \mu, \mu^{\prime}(f \cdot g \times f \cdot g)\right)=D\left((f \mu) \cdot(g \mu), \mu^{\prime}(f \times f) \cdot \mu^{\prime}(g \times g)\right) .
$$

On the other hand, since $\left[\mu^{\prime}(f \times f)\right]=\left[f p_{1}\right]\left[f p_{2}\right]$ and $[f \mu]$ are in the center, $D(f \mu$, $\left.\mu^{\prime}(f \times f)\right)$ is also in the center. Then, the following equations hold:

$$
\begin{aligned}
{[(f \mu) \cdot(g \mu)] } & =[f \mu][g \mu] \\
& =\left(D\left(f \mu, \mu^{\prime}(f \times f)\right)\left[\mu^{\prime}(f \times f)\right]\right)\left(D\left(g \mu, \mu^{\prime}(g \times g)\right)\left[\mu^{\prime}(g \times g)\right]\right) \\
& =\left(D\left(f \mu, \mu^{\prime}(f \times f)\right) D\left(g \mu, \mu^{\prime}(g \times g)\right)\right)\left[\mu^{\prime}(f \times f) \cdot \mu^{\prime}(g \times g)\right] .
\end{aligned}
$$

Therefore, $D\left((f \cdot g) \mu, \mu^{\prime}(f \cdot g \times f \cdot g)\right)=D\left(f \mu, \mu^{\prime}(f \times f)\right) D\left(g \mu, \mu^{\prime}(g \times g)\right)$.

COROLlary 2.12. [5, Corollary 2.4] If $Y$ is a homotopy associative and homotopy commutative H-space, then the H-deviation map is an algebraic loop homomorphism.

Proof. Since any maps from $X$ to $Y$ satisfy the condition of Lemma 2.10, it follows the assertion.

Definition 2.13. [5] Two central H-extensions

$$
\left(X, \mu_{X}\right) \stackrel{f_{i}}{\rightarrow}\left(Z_{i}, \mu_{i}\right) \stackrel{g_{i}}{\rightarrow}\left(Y, \mu_{Y}\right) \quad(i=1,2)
$$

are said to be equivalent if there exists an H-map $h:\left(Z_{1}, \mu_{1}\right) \rightarrow\left(Z_{2}, \mu_{2}\right)$ such that the following diagram is homotopy commutative.

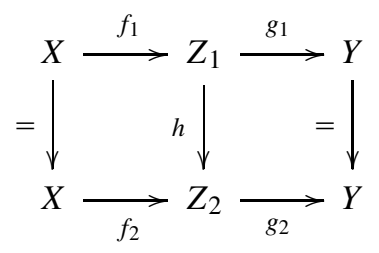

It is readily seen that this relation is an equivalence relation. We denote by $C H\left(X_{1}, \mu_{1} ; X_{2}, \mu_{2}\right)$ the set of equivalence classes of central H-extensions of $\left(X_{1}, \mu_{1}\right)$ by $\left(X_{2}, \mu_{2}\right)$, and denote by $\left[(X, \mu), f_{1}, f_{2}\right]$ the equivalence class of the central H-extension

$$
\left(X_{1}, \mu_{1}\right) \stackrel{f_{1}}{\rightarrow}(X, \mu) \stackrel{f_{2}}{\rightarrow}\left(X_{2}, \mu_{2}\right)
$$

THEOREM 2.14. [5, Theorem 4.3] Let $\left(X_{1}, \mu_{1}\right)$ be a homotopy associative and homotopy commutative $H$-space and let $\left(\mathrm{X}_{2}, \mu_{2}\right)$ be an $H$-space. Define

$$
\Phi:\left[X_{2} \wedge X_{2}, X_{1}\right] / \operatorname{Im} H D \longrightarrow C H\left(X_{1}, \mu_{1} ; X_{2}, \mu_{2}\right)
$$

by $\Phi([\omega])=\left[\left(X_{1} \times X_{2}, \mu_{\omega}\right), i_{1}, p_{2}\right]$, where the multiplication $\mu_{\omega}$ of $X_{1} \times X_{2}$ is defined by

$$
\mu_{\omega}\left(\left(x_{1}, x_{2}\right),\left(y_{1}, y_{2}\right)\right)=\left(\mu_{1}\left(\mu_{1}\left(x_{1}, y_{1}\right), \omega q\left(x_{2}, y_{2}\right)\right), \mu_{2}\left(x_{2}, y_{2}\right)\right),
$$

where $q: X_{2} \times X_{2} \rightarrow X_{2} \wedge X_{2}$ is the quotient map. Then $\Phi$ is bijective. 
We now check that $\left(X_{1} \times X_{2}, \mu_{\omega}\right)$ is a central H-extension of $X_{1}$ by $X_{2}$. It is easily seen that, for any space $Y$, the sequence of algebraic loops

$$
e \longrightarrow\left[Y, X_{1}\right] \stackrel{i_{1 *}}{\longrightarrow}\left[Y, X_{1} \times X_{2}\right] \stackrel{p_{2 *}}{\longrightarrow}\left[Y, X_{2}\right] \longrightarrow e
$$

is exact. For any $[f] \in\left[Y, X_{1}\right],[g],[h] \in\left[Y, X_{1} \times X_{2}\right]$ and $y \in Y$, we put $f_{1}:=f(y)$, $g_{i}:=p_{i} g(y)$ and $h_{i}:=p_{i} h(y)$ for convenience. Then, we have

$$
\begin{aligned}
\mu_{\omega}\left(g \times i_{1} f\right) \Delta(y) & =\left(\mu_{1}\left(\mu_{1}\left(g_{1}, f_{1}\right), \omega q\left(g_{2}, *\right)\right), \mu_{2}\left(g_{2}, *\right)\right) \\
& \simeq\left(\mu_{1}\left(\mu_{1}\left(f_{1}, g_{1}\right), \omega q\left(*, g_{2}\right)\right), \mu_{2}\left(*, g_{2}\right)\right)=\mu_{\omega}\left(i_{1} f \times g\right) \Delta(y)
\end{aligned}
$$

and

$$
\begin{aligned}
\mu_{\omega} & \left(\mu_{\omega}(g \times h) \Delta \times i_{1} f\right) \Delta(y) \\
& =\left(\mu_{1}\left(\mu_{1}\left(\mu_{1}\left(\mu_{1}\left(g_{1}, h_{1}\right), \omega q\left(g_{2}, h_{2}\right)\right), f_{1}\right), \omega q\left(\mu_{2}\left(g_{2}, h_{2}\right), *\right)\right), \mu_{2}\left(\mu_{2}\left(g_{2}, h_{2}\right), *\right)\right) \\
& \simeq\left(\mu_{1}\left(\mu_{1}\left(g_{1}, \mu_{1}\left(\mu_{1}\left(h_{1}, f_{1}\right), \omega q\left(h_{2}, *\right)\right), \omega q\left(g_{2}, h_{2}\right)\right)\right), \mu_{2}\left(g_{2}, \mu_{2}\left(h_{2}, *\right)\right)\right) \\
& =\mu_{\omega}\left(g \times \mu_{\omega}\left(h \times i_{1} f\right) \Delta\right) \Delta(y) .
\end{aligned}
$$

Thus, the equations $\left[i_{1} f\right][g]=[g]\left[i_{1} f\right]$ and $([g][h])\left[i_{1} f\right]=[g]\left([h]\left[i_{1} f\right]\right)$ hold. A similar argument shows that $\left[i_{1} f\right]([g][h])=\left(\left[i_{1} f\right][g]\right)[h]$. Therefore, $\left(X_{1} \times X_{2}, \mu_{\omega}\right)$ is a central H-extension.

Remark 2.15. We recall the inverse map of $\Phi, \Phi^{\prime}$, defined in [5, p. 256]. For any central H-extension $\left[(X, \mu), f_{1}, f_{2}\right]$ of $X_{1}$ by $X_{2}$, the exact sequence

$$
e \longrightarrow\left[X_{2}, X_{1}\right] \stackrel{f_{1 *}}{\longrightarrow}\left[X_{2}, X\right] \stackrel{f_{2 *}}{\longrightarrow}\left[X_{2}, X_{2}\right] \longrightarrow e
$$

shows that there is a map $g: X_{2} \rightarrow X$ such that $f_{2} g \simeq i d_{X_{2}}$. We consider the exact sequence

$$
e \longrightarrow\left[X_{2} \wedge X_{2}, X_{1}\right] \stackrel{f_{1 *}}{\longrightarrow}\left[X_{2} \wedge X_{2}, X\right] \stackrel{f_{2 *}}{\longrightarrow}\left[X_{2} \wedge X_{2}, X_{2}\right] \longrightarrow e .
$$

Since $f_{2 *}(H D(g))=H D\left(f_{2} g\right)=0$ in $\left[X_{2} \wedge X_{2}, X_{2}\right]$, there exists $\theta \in\left[X_{2} \wedge X_{2}, X_{1}\right]$ such that $f_{1 *}(\theta)=H D(g)$. Then, we define a map

$$
\Phi^{\prime}: C H\left(X_{1}, \mu_{1} ; X_{2}, \mu_{2}\right) \longrightarrow\left[X_{2} \wedge X_{2}, X_{1}\right] / \operatorname{Im} H D
$$

by $\Phi^{\prime}\left(\left[(X, \mu), f_{1}, f_{2}\right]\right)=[-\theta]$.

We note that, in [5, Theorem 4.3], Kachi defined the map $\bar{\Phi}$ from $\left[X_{2} \wedge X_{2}, X_{1}\right] / \operatorname{Im} H D$ to $C H\left(X_{1}, \mu_{1} ; X_{2}, \mu_{2}\right)$ by sending $[\omega]$ to $\left[\left(X_{1} \times X_{2}, \mu_{-\omega}\right), i_{1}, p_{2}\right]$ and claimed that this map is bijective and $\Phi^{\prime}$ is an inverse map of $\bar{\Phi}$. However, this map is not bijective since $\Phi^{\prime} \Phi([\omega])=-[\omega]$. We hence need to modify the definition of $\bar{\Phi}$ a little and we see that $\Phi$ is bijective and $\Phi^{\prime}$ is its inverse map using an argument similar to [5, Theorem 4.3]. Then combining the reason mentioned above and Remark 2.11, Theorem 4.3 in [5] is true without changing the proof.

By Theorem 2.14, for an equivalence class $\mathcal{E}$ in $C H\left(X_{1}, \mu_{1} ; X_{2}, \mu_{2}\right)$, there exists a map $\omega: X_{2} \wedge X_{2} \rightarrow X_{1}$ such that $\Phi([\omega])=[\mathcal{E}]$, the map $\omega$ is called the classifying map of the central H-extensions $\mathcal{E}$. Moreover, we refer to the set $\left[X_{2} \wedge X_{2}, X_{1}\right] / \operatorname{Im} H D$ as the moduli set of H-extensions of $\left(X_{1}, \mu_{1}\right)$ by $\left(X_{2}, \mu_{2}\right)$. 


\section{Moduli subsets of central H-extensions}

We retain the notation and terminology described in the previous section. Let $\left(X_{1}, \mu_{1}\right)$ be a homotopy associative and homotopy commutative $\mathrm{H}$-space, and let $\left(X_{2}, \mu_{2}\right)$ be an $\mathrm{H}$-space. Then, by Theorem 2.14 , a central H-extension of $\left(X_{1}, \mu_{1}\right)$ by $\left(X_{2}, \mu_{2}\right)$ is of the form $\left(X_{1} \times X_{2}, \mu_{\omega}\right)$. Let $i_{j}: X_{j} \rightarrow X_{1} \times X_{2}$ and $p_{j}: X_{1} \times X_{2} \rightarrow X_{j}$ denote the inclusions and the projections, respectively. Let $\Delta_{j}: X_{j} \rightarrow X_{j} \times X_{j}$ and $\Delta: X_{1} \times X_{2} \rightarrow\left(X_{1} \times X_{2}\right)^{2}$ be diagonal maps. We denote by $i d_{j}$ and $i d$ the identity maps of $X_{j}$ and $X_{1} \times X_{2}$, respectively. For convenience, we put $x x^{\prime}:=\mu_{1}\left(x, x^{\prime}\right)$ and $y y^{\prime}:=\mu_{2}\left(y, y^{\prime}\right)$ for any $x, x^{\prime} \in X_{1}$ and $y, y^{\prime} \in X_{2}$.

Proposition 3.1. The central H-extension $\left(X_{1} \times X_{2}, \mu_{\omega}\right)$ is inversive if and only if $\left(X_{2}, \mu_{2}\right)$ is inversive and $\omega q\left(l_{2} \times i d_{2}\right) \Delta_{2} \simeq \omega q\left(i d_{2} \times r_{2}\right) \Delta_{2}$, where $l_{2}$ and $r_{2}$ are the left inverse and the right inverse of $\left(X_{2}, \mu_{2}\right)$, respectively.

Before proving Proposition 3.1, we give the following lemmas.

LEMMA 3.2. Let $(X, \mu),\left(Y, \mu^{\prime}\right)$ be $H$-spaces and let $f: X \rightarrow Y$ be a map. If $f$ is an H-map, then $f l \simeq l^{\prime} f, f r \simeq r^{\prime} f$, where $l, l^{\prime}$ are left inverses of $(X, \mu),\left(Y, \mu^{\prime}\right)$ and $r, r^{\prime}$ are right inverses of $(X, \mu),\left(Y, \mu^{\prime}\right)$, respectively.

Proof. We see that $[f l][f]=[*]=\left[l^{\prime} f\right][f]$ in $[X, Y]$ since $f$ is an H-map. Hence, we get $[f l]=\left[l^{\prime} f\right]$. Similarly, one obtains the equality $[f r]=\left[r^{\prime} f\right]$.

LEMMA 3.3. Let $Y, Z$ be spaces and let $i: Y \rightarrow Y \times Z, p: Y \times Z \rightarrow Y$ be the inclusion and the projection. Then one has

$$
\operatorname{Ker}\left\{i^{*}:[Y \times Z, X] \rightarrow[Y, X]\right\} \cap \operatorname{Im}\left\{p^{*}:[Y, X] \rightarrow[Y \times Z, X]\right\}=\{e\}
$$

for any $H$-space $X$.

Proof. For any $[f] \in \operatorname{Ker} i^{*} \cap \operatorname{Im} p^{*}$, there is a map $g: Y \rightarrow X$ such that $f \simeq g q$. Since $[f]$ is in Ker $i^{*}$, it follows that $* \simeq f i \simeq g$ and hence $f \simeq *$.

Proof of Proposition 3.1. Let $l$ and $r$ be a left inverse and a right inverse of $\left(X_{1} \times X_{2}, \mu_{\omega}\right)$, respectively. Note that $l \simeq r$ if and only if $p_{j} l \simeq p_{j} r$ for $j=1,2$. In order to prove Proposition 3.1, it is enough to show

(1) $\quad p_{1} l \simeq p_{1} r$ if and only if $\omega q\left(l_{2} \times i d_{2}\right) \Delta_{2} \simeq \omega q\left(i d_{2} \times r_{2}\right) \Delta_{2}$ and

(2) $\quad p_{2} l \simeq p_{2} r$ if and only if $\left(X_{2}, \mu_{2}\right)$ is inversive.

Since

$$
* \simeq p_{1} \mu_{\omega}(l \times i d) \Delta=\mu_{1}\left(\mu_{1}\left(p_{1} l \times p_{1}\right) \Delta \times \omega q\left(p_{2} l \times p_{2}\right) \Delta\right) \Delta,
$$

it follows that

$$
[*]=\left(\left[p_{1} l\right]\left[p_{1}\right]\right)\left[\omega q\left(p_{2} l \times p_{2}\right) \Delta\right]
$$

in $\left[X_{1} \times X_{2}, X_{1}\right]$. Similarly, we have

$$
[*]=\left(\left[p_{1}\right]\left[p_{1} r\right]\right)\left[\omega q\left(p_{2} \times p_{2} r\right) \Delta\right]
$$

in $\left[X_{1} \times X_{2}, X_{1}\right]$. Since $\left[X_{1} \times X_{2}, X_{1}\right]$ is an abelian group, we see that $\left[p_{1} l\right]=\left[p_{1} r\right]$ if and only if $\left[\omega q\left(p_{2} l \times p_{2}\right) \Delta\right]=\left[\omega q\left(p_{2} \times p_{2} r\right) \Delta\right]$. Lemma 3.2 allows us to obtain that

$$
\left[\omega q\left(p_{2} l \times p_{2}\right) \Delta\right]=\left[\omega q\left(l_{2} \times i d_{2}\right) \Delta_{2} p_{2}\right]
$$


and

$$
\left[\omega q\left(p_{2} \times p_{2} r\right) \Delta\right]=\left[\omega q\left(i d_{2} \times r_{2}\right) \Delta_{2} p_{2}\right] .
$$

If $\left[\omega q\left(l_{2} \times i d_{2}\right) \Delta_{2} p_{2}\right]=\left[\omega q\left(i d_{2} \times r_{2}\right) \Delta_{2} p_{2}\right]$, then

$$
\left[\omega q\left(l_{2} \times i d_{2}\right) \Delta_{2}\right]=\left[\omega q\left(l_{2} \times i d_{2}\right) \Delta_{2} p_{2} i_{2}\right]=\left[\omega q\left(i d_{2} \times r_{2}\right) \Delta_{2} p_{2} i_{2}\right]=\left[\omega q\left(i d_{2} \times r_{2}\right) \Delta_{2}\right] .
$$

Therefore we have assertion (1).

Suppose that $p_{2} l \simeq p_{2} r$. Then $l_{2} \simeq p_{2} l i_{2} \simeq p_{2} r i_{2} \simeq r_{2}$; that is, $\left(X_{2}, \mu_{2}\right)$ is inversive. Conversely, suppose that $\left(X_{2}, \mu_{2}\right)$ is inversive. Then it follows that $p_{2} l i_{2} \simeq p_{2} r i_{2}$ and hence

$$
[*]=\left[p_{2} l i_{2}\right]\left[p_{2} r i_{2}\right]^{-1}=\left[p_{2} l i_{2}\right]\left[r_{2} p_{2} r i_{2}\right]=i_{2}^{*}\left(\left[p_{2} l\right]\left[r_{2} p_{2} r\right]\right) .
$$

Therefore we see that $\left[p_{2} l\right]\left[r_{2} p_{2} r\right] \in \operatorname{Ker} i_{2}^{*}$. On the other hand,

$$
\left[p_{2} l\right]\left[r_{2} p_{2} r\right]=\left[l_{2} p_{2}\right]\left[r_{2} r_{2} p_{2}\right]=p_{2}^{*}\left(\left[l_{2}\right]\left[r_{2} r_{2}\right]\right) \in \operatorname{Im} p_{2}^{*} .
$$

In view of Lemma 3.3, we have

$$
[*]=\left[p_{2} l\right]\left[r_{2} p_{2} r\right]=\left[p_{2} l\right]\left[p_{2} r\right]^{-1} .
$$

Hence, it follows that $\left[p_{2} l\right]=\left[p_{2} r\right]$. We have the second assertion.

Proposition 3.4. The central H-extension $\left(X_{1} \times X_{2}, \mu_{\omega}\right)$ is power associative if and only if $\left(X_{2}, \mu_{2}\right)$ is power associative and $\omega q\left(\mu_{2} \times i d_{2}\right) \bar{\Delta}_{2} \simeq \omega q\left(i d_{2} \times \mu_{2}\right) \bar{\Delta}_{2}$, where $\bar{\Delta}_{2}=$ $\left(\Delta_{2} \times i d_{2}\right) \Delta_{2}$.

Proof. As in the proof of Proposition 3.1, it is enough to show the following:

(1) $p_{1} \mu_{\omega}\left(\mu_{\omega} \times i d\right) \bar{\Delta} \simeq p_{1} \mu_{\omega}\left(i d \times \mu_{\omega}\right) \bar{\Delta}$ if and only if $\omega q\left(\mu_{2} \times i d_{2}\right) \bar{\Delta}_{2} \simeq \omega q\left(i d_{2} \times\right.$ $\left.\mu_{2}\right) \bar{\Delta}_{2}$, where $\bar{\Delta}=(\Delta \times i d) \Delta$;

(2) $\quad p_{2} \mu_{\omega}\left(\mu_{\omega} \times i d\right) \bar{\Delta} \simeq p_{2} \mu_{\omega}\left(i d \times \mu_{\omega}\right) \bar{\Delta}$ if and only if $\left(X_{2}, \mu_{2}\right)$ is power associative.

Given $(x, y) \in X_{1} \times X_{2}$, then, we have

$$
\begin{aligned}
\mu_{\omega}\left(\mu_{\omega} \times i d\right) \bar{\Delta}(x, y) & =\mu_{\omega}(((x x) \omega q(y, y), y y),(x, y)) \\
& =((((x x) \omega q(y, y)) x) \omega q(y y, y),(y y) y)
\end{aligned}
$$

and

$$
\begin{aligned}
\mu_{\omega}\left(i d \times \mu_{\omega}\right) \bar{\Delta}(x, y) & =\mu_{\omega}((x, y),((x x) \omega q(y, y), y y)) \\
& =((x((x x) \omega q(y, y))) \omega q(y, y y), y(y y)) .
\end{aligned}
$$

Hence, assertions (1) and (2) follow from the equations.

The same argument as in Proposition 3.4 does work to prove the following propositions.

Proposition 3.5. The central H-extension $\left(X_{1} \times X_{2}, \mu_{\omega}\right)$ is Moufang if and only if $\left(X_{2}, \mu_{2}\right)$ is Moufang and

$$
\begin{aligned}
& {\left[\mu_{1}(\omega q \times \omega q) \theta_{2}\right]\left[\omega q\left(\mu_{2} \times \mu_{2}\right) \theta_{2}\right]} \\
& \quad=\left[\mu_{1}(\omega q \times \omega q)\left(i d_{2} \times\left(\mu_{2} \times i d_{2} \times i d_{2}\right) \Delta_{2}^{\prime}\right)\right]\left[\omega q\left(\mu_{2}\left(i d_{2} \times \mu_{2}\right) \times i d_{2}\right) \theta_{2}\right]
\end{aligned}
$$

in $\left[X_{2}^{3}, X_{1}\right]$, where $\Delta_{2}^{\prime}$ is the diagonal map of $X_{2} \times X_{2}$ and $\theta_{2}: X_{2}^{3} \longrightarrow X_{2}^{4}$ is defined by $\theta_{2}(x, y, z)=(x, y, z, x)$. 
Proof. Let $\left(x_{i}, y_{i}\right) \in X_{1} \times X_{2}$ for $i=1,2,3$. The following equations hold:

$$
\begin{aligned}
\mu_{\omega} & \left(\mu_{\omega} \times \mu_{\omega}\right) \theta\left(\left(x_{1}, y_{1}\right),\left(x_{2}, y_{2}\right),\left(x_{3}, y_{3}\right)\right) \\
& =\mu_{\omega}\left(\left(\left(x_{1} x_{2}\right) \omega q\left(y_{1}, y_{2}\right), y_{1} y_{2}\right),\left(\left(x_{3} x_{1}\right) \omega q\left(y_{3}, y_{1}\right), y_{3} y_{1}\right)\right) \\
& =\left(\left(\left(\left(x_{1} x_{2}\right) \omega q\left(y_{1}, y_{2}\right)\right)\left(\left(x_{3} x_{1}\right) \omega q\left(y_{3}, y_{1}\right)\right)\right) \omega q\left(y_{1} y_{2}, y_{3} y_{1}\right),\left(y_{1} y_{2}\right)\left(y_{3} y_{1}\right)\right)
\end{aligned}
$$

and

$$
\begin{aligned}
& \mu_{\omega}\left(\mu_{\omega} \times i d\right)\left(i d \times \mu_{\omega} \times i d\right) \theta\left(\left(x_{1}, y_{1}\right),\left(x_{2}, y_{2}\right),\left(x_{3}, y_{3}\right)\right) \\
& \quad=\mu_{\omega}\left(\mu_{\omega} \times i d\right)\left(\left(x_{1}, y_{1}\right),\left(\left(x_{2} x_{3}\right) \omega q\left(y_{2}, y_{3}\right), y_{2} y_{3}\right),\left(x_{1}, y_{1}\right)\right) \\
& \quad=\mu_{\omega}\left(\left(\left(x_{1}\left(\left(x_{2} x_{3}\right) \omega q\left(y_{2}, y_{3}\right)\right)\right) \omega q\left(y_{1}, y_{2} y_{3}\right), y_{1}\left(y_{2} y_{3}\right)\right),\left(x_{1}, y_{1}\right)\right) \\
& \quad=\left(\left(\left(\left(x_{1}\left(\left(x_{2} x_{3}\right) \omega q\left(y_{2}, y_{3}\right)\right)\right) \omega q\left(y_{1}, y_{2} y_{3}\right)\right) x_{1}\right) \omega q\left(y_{1}\left(y_{2} y_{3}\right), y_{1}\right),\left(y_{1}\left(y_{2} y_{3}\right)\right) y_{1}\right) .
\end{aligned}
$$

These imply the assertion.

Proposition 3.6. The central H-extension $\left(X_{1} \times X_{2}, \mu_{\omega}\right)$ is flexible if and only if $\left(X_{2}, \mu_{2}\right)$ is flexible and

$$
\begin{aligned}
& \mu_{1}(\omega q \times \omega q)\left(t \times\left(i d_{2} \times \mu_{2} t\right)\left(\Delta_{2} \times i d_{2}\right)\right) \Delta_{2}^{\prime} \\
& \quad \simeq \mu_{1}(\omega q \times \omega q)\left(i d_{2} \times i d_{2} \times\left(\mu_{2} \times i d_{2}\right)\left(i d_{2} \times t\right)\left(\Delta_{2} \times i d_{2}\right)\right) \Delta_{2}^{\prime} .
\end{aligned}
$$

Proof. For any $\left(x_{1}, y_{1}\right),\left(x_{2}, y_{2}\right) \in X_{1} \times X_{2}$, we have

$$
\begin{aligned}
\mu_{\omega} & \left(\mu_{\omega} \times i d\right)(i d \times t)(\Delta \times i d)\left(\left(x_{1}, y_{1}\right),\left(x_{2}, y_{2}\right)\right) \\
& =\mu_{\omega}\left(\left(\left(x_{1} x_{2}\right) \omega q\left(y_{1}, y_{2}\right), y_{1} y_{2}\right),\left(x_{1}, y_{1}\right)\right) \\
& =\left(\left(\left(\left(x_{1} x_{2}\right) \omega q\left(y_{1}, y_{2}\right)\right) x_{1}\right) \omega q\left(y_{1} y_{2}, y_{1}\right),\left(y_{1} y_{2}\right) y_{1}\right)
\end{aligned}
$$

and

$$
\begin{aligned}
\mu_{\omega} & \left(i d \times \mu_{\omega}\right)(i d \times t)(\Delta \times i d)\left(\left(x_{1}, y_{1}\right),\left(x_{2}, y_{2}\right)\right) \\
& =\mu_{\omega}\left(\left(x_{1}, y_{1}\right),\left(\left(x_{2} x_{1}\right) \omega q\left(y_{2}, y_{1}\right), y_{2} y_{1}\right)\right) \\
& =\left(\left(x_{1}\left(\left(x_{2} x_{1}\right) \omega q\left(y_{2}, y_{1}\right)\right)\right) \omega q\left(y_{1}, y_{2} y_{1}\right), y_{1}\left(y_{2} y_{1}\right)\right) .
\end{aligned}
$$

Therefore, we obtain the result.

Next, we consider the following subsets of the homotopy set $\left[X_{2} \wedge X_{2}, X_{1}\right]$ :

$$
\begin{aligned}
G_{\mathrm{inv}} & =\left\{[\omega] \in\left[X_{2} \wedge X_{2}, X_{1}\right] \mid \omega q\left(l_{2} \times i d_{2}\right) \Delta_{2} \simeq \omega q\left(i d_{2} \times r_{2}\right) \Delta_{2}\right\}, \\
G_{\mathrm{p} . \mathrm{a}} & =\left\{[\omega] \in\left[X_{2} \wedge X_{2}, X_{1}\right] \mid \omega q\left(\mu_{2} \times i d_{2}\right) \bar{\Delta}_{2} \simeq \omega q\left(i d_{2} \times \mu_{2}\right) \bar{\Delta}_{2}\right\}, \\
G_{\mathrm{Mo}} & =\left\{[\omega] \in\left[X_{2} \wedge X_{2}, X_{1}\right] \mid \Gamma_{\mathrm{Mo}}(\omega) \simeq \Gamma_{\mathrm{Mo}}^{\prime}(\omega)\right\}, \\
G_{\mathrm{fle}} & =\left\{[\omega] \in\left[X_{2} \wedge X_{2}, X_{1}\right] \mid \mu_{1}(\omega \times \omega) \Gamma_{\mathrm{fle}} \simeq \mu_{1}(\omega \times \omega) \Gamma_{\mathrm{fle}}^{\prime}\right\},
\end{aligned}
$$

where

$$
\begin{aligned}
\Gamma_{\mathrm{Mo}}(\omega) & =\mu_{1}\left(\mu_{1}(\omega q \times \omega q) \theta_{2} \times \omega q\left(\mu_{2} \times \mu_{2}\right) \theta_{2}\right) \Delta_{X_{2}^{3}}, \\
\Gamma_{\mathrm{Mo}}^{\prime}(\omega) & =\mu_{1}\left(\mu_{1}(\omega q \times \omega q)\left(i d_{2} \times\left(\mu_{2} \times i d_{X_{2}^{2}}\right) \Delta_{2}^{\prime}\right) \times \omega q\left(\mu_{2}\left(i d_{2} \times \mu_{2}\right) \times i d_{2}\right) \theta_{2}\right) \Delta_{X_{2}^{3}}, \\
\Gamma_{\mathrm{fle}} & =(q \times q)\left(t \times\left(i d_{2} \times \mu_{2} t\right)\left(\Delta_{2} \times i d_{2}\right)\right) \Delta_{2}^{\prime}, \\
\Gamma_{\mathrm{fle}}^{\prime} & =(q \times q)\left(i d_{2} \times i d_{2} \times\left(\mu_{2} \times i d_{2}\right)\left(i d_{2} \times t\right)\left(\Delta_{2} \times i d_{2}\right)\right) \Delta_{2}^{\prime} .
\end{aligned}
$$


LeMmA 3.7. The sets $G_{\mathrm{inv}}, G_{\mathrm{p} . \mathrm{a}}, G_{\mathrm{Mo}}$ and $G_{\mathrm{fle}}$ are subgroups of $\left[X_{2} \wedge X_{2}, X_{1}\right]$.

Proof. For any $\left[\omega_{1}\right],\left[\omega_{2}\right] \in G_{\text {inv }}$, we have

$$
\begin{aligned}
{\left[\mu_{1}\left(\omega_{1} \times \omega_{2}\right) \Delta_{2}^{\prime \prime} q\left(l_{2} \times i d_{2}\right) \Delta_{2}\right] } & =\left[\omega_{1} q\left(l_{2} \times i d_{2}\right) \Delta_{2}\right]\left[\omega_{2} q\left(l_{2} \times i d_{2}\right) \Delta_{2}\right] \\
& =\left[\omega_{1} q\left(i d_{2} \times r_{2}\right) \Delta_{2}\right]\left[\omega_{2} q\left(i d_{2} \times r_{2}\right) \Delta_{2}\right] \\
& =\left[\mu_{1}\left(\omega_{1} \times \omega_{2}\right) \Delta_{2}^{\prime \prime} q\left(i d_{2} \times r_{2}\right) \Delta_{2}\right]
\end{aligned}
$$

where $\Delta_{2}^{\prime \prime}$ is the diagonal map of $X_{2} \wedge X_{2}$. Hence $\left[\omega_{1}\right]\left[\omega_{2}\right] \in G_{\text {inv }}$. Let $l_{1}$ be a left inverse of $\left(X_{1}, \mu_{1}\right)$. Then we have

$$
\left[l_{1} \omega_{1} q\left(l_{2} \times i d_{2}\right) \Delta_{2}\right]=\left[l_{1} \omega_{1} q\left(i d_{2} \times r_{2}\right) \Delta_{2}\right] .
$$

Hence we obtain $\left[\omega_{1}\right]^{-1}=\left[l_{1} \omega_{1}\right] \in G_{\text {inv }}$. Similarly, we can show that $G_{\text {p.a }}, G_{\text {Mo }}$ and $G_{\text {fle }}$ are subgroups of $\left[X_{2} \wedge X_{2}, X_{1}\right]$.

Let $\Phi:\left[X_{2} \wedge X_{2}, X_{1}\right] / \operatorname{Im} H D \rightarrow C H\left(X_{1}, \mu_{1} ; X_{2}, \mu_{2}\right)$ be the bijection mentioned in Theorem 2.14.

LEMMA 3.8. If $\left(X_{2}, \mu_{2}\right)$ is inversive (respectively, power associative, Moufang and flexible), then $\operatorname{Im} H D \subset G_{\mathrm{inv}}$ (respectively, $\operatorname{Im} H D \subset G_{\mathrm{p} . \mathrm{a}}, \operatorname{Im} H D \subset G_{\mathrm{Mo}}$ and $\operatorname{Im} H D \subset$ $\left.G_{\text {fle }}\right)$.

Proof. For any $[\omega] \in \operatorname{Im} H D, \Phi([\omega])=\left[\left(X_{1} \times X_{2}, \mu_{1} \times \mu_{2}\right), i_{1}, p_{2}\right]$. Since $\left(X_{2}, \mu_{2}\right)$ is inversive, $\left(X_{1} \times X_{2}, \mu_{1} \times \mu_{2}\right)$ is also inversive, and hence $\omega q\left(l_{2} \times i d_{2}\right) \Delta_{2} \simeq \omega q\left(i d_{2} \times\right.$ $\left.r_{2}\right) \Delta_{2}$ by Proposition 3.1. We thus see that $[\omega] \in G_{\text {inv }}$. A similar augment shows the other cases.

THEOREM 3.9. The following statements hold.

(1) If $\left(X_{2}, \mu_{2}\right)$ is inversive, then

$\Phi_{\text {inv }}: G_{\text {inv }} / \operatorname{Im} H D \rightarrow\left\{\left[(X, \mu), f_{1}, f_{2}\right] \in C H\left(X_{1}, \mu_{1} ; X_{2}, \mu_{2}\right) \mid(X, \mu)\right.$ is inversive $\}$,

which is the restricted homomorphism of $\Phi$ to $G_{\mathrm{inv}} / \mathrm{Im} H D$, is bijective.

(2) If $\left(X_{2}, \mu_{2}\right)$ is power associative, then

$$
\begin{aligned}
\Phi_{\mathrm{p} . \mathrm{a}}: G_{\mathrm{p} . \mathrm{a}} / \operatorname{Im} H D \rightarrow & \left\{\left[(X, \mu), f_{1}, f_{2}\right] \in C H\left(X_{1}, \mu_{1} ; X_{2}, \mu_{2}\right) \mid\right. \\
& (X, \mu) \text { is power associative }\},
\end{aligned}
$$

which is the restricted homomorphism of $\Phi$ to $G_{\mathrm{p} . \mathrm{a}} / \mathrm{Im} H D$, is bijective.

(3) If $\left(X_{2}, \mu_{2}\right)$ is Moufang, then

$\Phi_{\mathrm{Mo}}: G_{\mathrm{Mo}} / \operatorname{Im} H D \rightarrow\left\{\left[(X, \mu), f_{1}, f_{2}\right] \in C H\left(X_{1}, \mu_{1} ; X_{2}, \mu_{2}\right) \mid(X, \mu)\right.$ is Moufang $\}$, which is the restricted homomorphism of $\Phi$ to $G_{\mathrm{Mo}} / \mathrm{Im} H D$, is bijective.

(4) If $\left(X_{2}, \mu_{2}\right)$ is flexible, then

$$
\Phi_{\mathrm{fle}}: G_{\mathrm{fle}} / \operatorname{Im} H D \rightarrow\left\{\left[(X, \mu), f_{1}, f_{2}\right] \in C H\left(X_{1}, \mu_{1} ; X_{2}, \mu_{2}\right) \mid(X, \mu) \text { is flexible }\right\},
$$

which is the restricted homomorphism of $\Phi$ to $G_{\mathrm{fle}} / \mathrm{Im} H D$, is bijective. 
Proof. By Proposition 3.1, we see that the map $\Phi_{\text {inv }}$ is a well-defined map and hence it is injective. Let $(X, \mu)$ be an inversive central H-extension of $\left(X_{1}, \mu_{1}\right)$ by $\left(X_{2}, \mu_{2}\right)$. Theorem 2.14 yields that there exists a map $\omega: X_{2} \wedge X_{2} \rightarrow X_{1}$ such that $\left[(X, \mu), f_{1}, f_{2}\right]=$ $\left[\left(X_{1} \times X_{2}, \mu_{\omega}\right), i_{1}, p_{2}\right]$. Since $(X, \mu)$ is inversive, $\left(X_{1} \times X_{2}, \mu_{\omega}\right)$ is also inversive, and hence $\omega q\left(l_{2} \times i d_{2}\right) \Delta_{2} \simeq \omega q\left(i d_{2} \times r_{2}\right) \Delta_{2}$ by Proposition 3.1. Therefore $\Phi_{\text {inv }}([\omega])=$ $\left[(X, \mu), f_{1}, f_{2}\right]$ and $\Phi_{\text {inv }}$ is surjective. In the same manner, we can show that $\Phi_{\text {p.a }}, \Phi_{\text {Mo }}$ and $\Phi_{\text {fle }}$ are bijective.

We denote $G_{\text {inv }} / \operatorname{Im} H D, G_{\text {p.a }} / \operatorname{Im} H D, G_{\text {Mo }} / \operatorname{Im} H D$ and $G_{\text {fle }} / \operatorname{Im} H D$ by $S_{\text {inv }}, S_{\text {p.a }}, S_{\text {Mo }}$ and $S_{\mathrm{fle}}$, respectively, and call them the moduli subset of the H-extensions associated with the corresponding properties.

\section{The moduli sets of central H-extensions of rational H-spaces}

In this section, we will investigate the moduli set $G / \operatorname{Im} H D$ and its subset $S_{\text {inv }}, S_{\text {p.a }}, S_{\text {Mo }}$ and $S_{\text {fle }}$ in the rational cases. If $(X, \mu)$ is a $\mathbb{Q}$-local, simply-connected H-space and the homotopy group $\pi_{*}(X)$ are of finite type, then, by $[10, \S 8$, Theorem 12$], H^{*}(X ; \mathbb{Q}) \cong$ $\bigotimes_{i} \mathbb{Q}\left[x_{i}\right] \otimes \bigotimes_{j} \Lambda\left(y_{j}\right)$ as an algebra, where $\mathbb{Q}\left[x_{i}\right]$ is a polynomial algebra and $\Lambda\left(y_{j}\right)$ is an exterior algebra.

TheOREM 4.1. [8, Proposition 1] Let $(X, \mu)$ be a $\mathbb{Q}$-local, simply-connected H-space where $\pi_{*}(X)$ are of finite type, and let $Y$ be a space. Then the canonical map

$$
[Y, X] \longrightarrow \operatorname{Hom}_{\mathrm{Alg}}\left(H^{*}(X ; \mathbb{Q}), H^{*}(Y ; \mathbb{Q})\right), f \longmapsto H^{*}(f)
$$

is bijective.

According to Arkowitz and Lupton $[1]$, we give $\operatorname{Hom}_{\mathrm{Alg}}\left(H^{*}(X ; \mathbb{Q}), H^{*}(Y ; \mathbb{Q})\right)$ an algebraic loop structure so that the above canonical map is an algebraic loop homomorphism.

Definition 4.2. [1, Definition 2.1] Let $M$ be a free commutative graded algebra over $\mathbb{Q}$ of the form $\bigotimes_{i} \mathbb{Q}\left[x_{i}\right] \otimes \bigotimes_{j} \Lambda\left(y_{j}\right)$. A homomorphism $v: M \rightarrow M \otimes M$ is called $a$ diagonal if the following diagram is commutative, where $\varepsilon: M \rightarrow \mathbb{Q}$ is the augmentation.

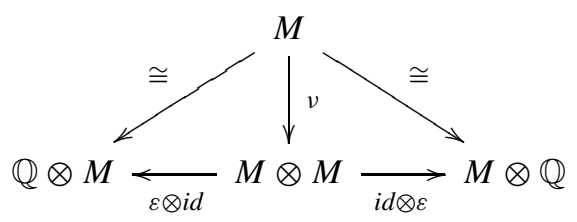

We note that, for any H-space $(X, \mu), H^{*}(\mu): H^{*}(X ; \mathbb{Q}) \rightarrow H^{*}(X ; \mathbb{Q}) \otimes H^{*}(X ; \mathbb{Q})$ is a diagonal.

THEOREM 4.3. [1, Lemma 3.1] Let $M=\bigotimes_{i} \mathbb{Q}\left[x_{i}\right] \otimes \bigotimes_{j} \Lambda\left(y_{j}\right)$ with the diagonal map $v$ : $M \rightarrow M \otimes M$, and let $A$ be a graded algebra. We define the product of $\operatorname{Hom}_{\mathrm{Alg}}(M, A)$ by

$$
\alpha \cdot \beta=m(\alpha \otimes \beta) \nu,
$$

where $m$ is the product of $A$. Then $\operatorname{Hom}_{\mathrm{Alg}}(M, A)$ is an algebraic loop endowed with the product. 
Let $\gamma_{1}$ and $\gamma_{2}$ be the elements in $\operatorname{Hom}_{\mathrm{Alg}}(M, A)$ such that $\gamma_{1} \cdot \alpha=\beta$ and $\alpha \cdot \gamma_{2}=\beta$. Then, by the proof of Theorem 4.3, the following equations hold:

$$
\begin{aligned}
& \gamma_{1}(z)=\beta(z)-\alpha(z)-m\left(\gamma_{1} \otimes \alpha\right) P(z), \\
& \gamma_{2}(z)=\beta(z)-\alpha(z)-m\left(\alpha \otimes \gamma_{2}\right) P(z),
\end{aligned}
$$

where $z$ is a generator of $M$ and $P(z)=v(z)-z \otimes 1-1 \otimes z$.

Definition 4.4. A left inverse $\lambda$ (respectively, a right inverse $\rho$ ) of an algebraic loop $\operatorname{Hom}_{\mathrm{Alg}}(M, M)$ is an element of $\operatorname{Hom}_{\mathrm{Alg}}(M, M)$ such that $\lambda \cdot i d=e$ (respectively, $i d \cdot \rho$ $=e$ ), where $e$ is the unit of $\operatorname{Hom}_{\mathrm{Alg}}(M, M)$.

Let $A$ be a graded algebra and let $M$ be a free commutative graded algebra of the form $\bigotimes_{i} \mathbb{Q}\left[x_{i}\right] \otimes \bigotimes_{j} \Lambda\left(y_{j}\right)$ for which each $x_{i}$ and $y_{i}$ are primitive; that is, $v\left(x_{i}\right)=x_{i} \otimes 1+$ $1 \otimes x_{i}$ and $v\left(y_{j}\right)=y_{j} \otimes 1+1 \otimes y_{j}$. We consider the bijection of sets

$$
\Psi: \operatorname{Hom}_{\mathrm{Alg}}(M, A) \stackrel{\cong}{\rightarrow} \operatorname{Hom}_{\mathbb{Q}}\left(\mathbb{Q}\left\langle x_{i}, y_{j}\right\rangle, A\right)
$$

which is defined by

$$
\Psi(\alpha)\left(x_{i}\right)=\alpha\left(x_{i}\right), \quad \Psi(\alpha)\left(y_{i}\right)=\alpha\left(y_{i}\right) \quad \text { for } \alpha \in \operatorname{Hom}_{\mathrm{Alg}}(M, A),
$$

where $\mathbb{Q}\left\langle x_{i}, y_{j}\right\rangle$ denotes the graded $\mathbb{Q}$-vector space with basis $x_{i}$ and $y_{j}$ for any $i$ and $j$. Let $\operatorname{Hom}_{\mathbb{Q}}\left(\mathbb{Q}\left\langle x_{i}, y_{j}\right\rangle, A\right)$ denote the set of $\mathbb{Q}$-linear maps from $\mathbb{Q}\left\langle x_{i}, y_{j}\right\rangle$ to $A$. Since $\operatorname{Hom}_{\mathbb{Q}}\left(\mathbb{Q}\left\langle x_{i}, y_{j}\right\rangle, A\right)$ is a $\mathbb{Q}$-vector space with respect to the canonical sum and the canonical scalar multiple, we can give the $\mathbb{Q}$-vector space structure to $\operatorname{Hom}_{\mathrm{Alg}}(M, A)$ via $\Psi$. We regard $\operatorname{Hom}_{\mathbb{Q}}\left(\mathbb{Q}\left\langle x_{i}, y_{j}\right\rangle, A\right)$ as an algebraic loop with the canonical sum. Then $\Psi$ is a morphism of algebraic loops. In fact, for any basis $z$ of $\mathbb{Q}\left\langle x_{i}, y_{j}\right\rangle$,

$$
(\alpha \cdot \beta)(z)=m(\alpha \otimes \beta) v(z)=\alpha(z)+\beta(z)=(\alpha+\beta)(z) .
$$

Let $\left(X_{1}, \mu_{1}\right)$ be a $\mathbb{Q}$-local, simply-connected, homotopy associative and homotopy commutative $\mathrm{H}$-space and the homotopy group of $X_{1}$ are of finite type, let $\left(X_{2}, \mu_{2}\right)$ be an H-space, and let $H^{*}\left(X_{1} ; \mathbb{Q}\right)=\bigotimes_{i} \mathbb{Q}\left[x_{i}\right] \otimes \bigotimes_{j} \Lambda\left(y_{j}\right)$. Since $\left(X_{1}, \mu_{1}\right)$ is a homotopy associative and homotopy commutative $\mathrm{H}$-space, we see that each $x_{i}$ and $y_{j}$ are primitive; see [6, Corollary 4.18].

LEMMA 4.5. Let $X$ and $Y$ be spaces. Then, for any $n>0$, there is an isomorphism of vector spaces

$$
H^{n}(X \wedge Y ; \mathbb{Q}) \cong\left(H^{>0}(X ; \mathbb{Q}) \otimes H^{>0}(Y ; \mathbb{Q})\right)^{n} .
$$

Proof. For any $n>0$, we have the following commutative diagram with exact rows.

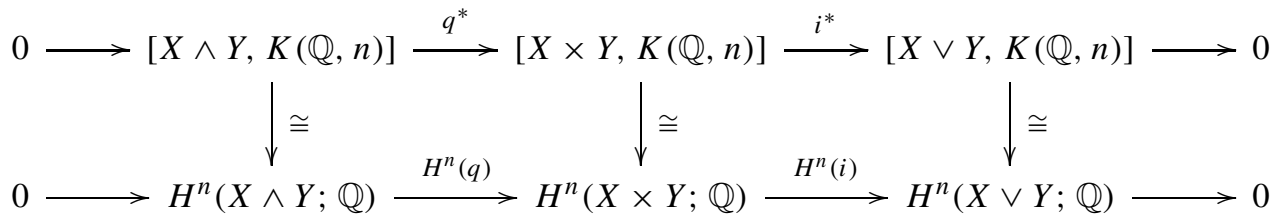

Then it follows that

$$
H^{n}(X \wedge Y ; \mathbb{Q}) \cong \operatorname{Ker} H^{n}(i)=\left(H^{>0}(X ; \mathbb{Q}) \otimes H^{>0}(Y ; \mathbb{Q})\right)^{n} .
$$


Define the map

$$
\overline{H D}: \operatorname{Hom}_{\mathbb{Q}}\left(\mathbb{Q}\left\langle x_{i}, y_{j}\right\rangle, H^{*}\left(X_{2} ; \mathbb{Q}\right)\right) \rightarrow \operatorname{Hom}_{\mathbb{Q}}\left(\mathbb{Q}\left\langle x_{i}, y_{j}\right\rangle, H^{*}\left(X_{2} \wedge X_{2} ; \mathbb{Q}\right)\right)
$$

by

$$
\overline{H D}(\alpha)(z)=P_{2}(\alpha(z)) \quad \text { for } \alpha \in \operatorname{Hom}_{\mathbb{Q}}\left(\mathbb{Q}\left\langle x_{i}, y_{j}\right\rangle, H^{*}\left(X_{2} ; \mathbb{Q}\right)\right),
$$

where $P_{2}(z)=H^{*}\left(\mu_{2}\right)(z)-z \otimes 1-1 \otimes z$ for any $z \in H^{*}\left(X_{2} ; \mathbb{Q}\right)$. Then we see that $\overline{H D}$ is a $\mathbb{Q}$-linear map.

PROPOSITION 4.6. The following diagram of algebraic loops is commutative.

$$
\begin{gathered}
\operatorname{Hom}_{\mathbb{Q}}\left(\mathbb{Q}\left\langle x_{i}, y_{j}\right\rangle, H^{*}\left(X_{2} ; \mathbb{Q}\right)\right) \stackrel{\overline{H D}}{\longrightarrow} \operatorname{Hom}_{\mathbb{Q}}\left(\mathbb{Q}\left\langle x_{i}, y_{j}\right\rangle, H^{*}\left(X_{2} \wedge X_{2} ; \mathbb{Q}\right)\right) \\
\Psi H^{*} \uparrow \cong \\
\quad \cong \uparrow \Psi H^{*} \\
{\left[X_{2}, X_{1}\right] \stackrel{H D}{\longrightarrow}\left[X_{2} \wedge X_{2}, X_{1}\right]}
\end{gathered}
$$

Proof. Using the isomorphism $\Psi$, we see that for any $f \in\left[X_{2}, X_{1}\right]$ there exists a unique element $\bar{D}(f) \in \operatorname{Hom}_{\mathbb{Q}}\left(\mathbb{Q}\left\langle x_{i}, y_{j}\right\rangle, H^{*}\left(X_{2} \times X_{2} ; \mathbb{Q}\right)\right)$ such that

$$
\bar{D}(f)+\left(H^{*}(f) \otimes H^{*}(f)\right) \Psi H^{*}\left(\mu_{1}\right)=H^{*}\left(\mu_{2}\right) \Psi H^{*}(f) .
$$

Therefore, it follows that $\bar{D}(f)=\Psi H^{*}\left(D\left(f \mu_{1}, \mu_{2}(f \times f)\right)\right)$. Observe that each $x_{i}$ and $y_{j}$ are primitive. In view of the proof of Theorem 4.3, we have

$$
\bar{D}(f)(z)=P_{2}\left(\Psi H^{*}(f)(z)\right)
$$

for any generators $z$ of $\mathbb{Q}\left\langle x_{i}, y_{j}\right\rangle$. Lemma 4.5 implies that

$$
\bar{D}(f)(z)=H^{*}(q) \overline{H D}\left(\Psi H^{*}(f)\right)(z) .
$$

Hence, by the definition of an H-deviation, we obtain

$$
H^{*}(q) \Psi H^{*}(H D(f))=\Psi H^{*}\left(D\left(f \mu_{1}, \mu_{2}(f \times f)\right)\right)=H^{*}(q) \overline{H D}\left(\Psi H^{*}(f)\right) .
$$

Since $H^{*}(q)$ is injective, it follows from Lemma 2.9 and Theorem 4.1 that $\Psi H^{*}(H D(f))=$ $\overline{H D}\left(\Psi H^{*}(f)\right)$.

$$
\begin{aligned}
\text { Put } V & =\operatorname{Hom}_{\mathbb{Q}}\left(\mathbb{Q}\left\langle x_{i}, y_{j}\right\rangle, H^{*}\left(X_{2} \wedge X_{2} ; \mathbb{Q}\right)\right) \text { and set } \\
V_{\mathrm{inv}} & =\left\{\alpha \in V \mid m_{2}\left(\lambda_{2} \otimes i d\right) H^{*}(q) \alpha=m_{2}\left(i d \otimes \rho_{2}\right) H^{*}(q) \alpha\right\}, \\
V_{\mathrm{p} . \mathrm{a}} & =\left\{\alpha \in V \mid \bar{m}_{2}\left(H^{*}\left(\mu_{2}\right) \otimes i d\right) H^{*}(q) \alpha=\bar{m}_{2}\left(i d \otimes H^{*}\left(\mu_{2}\right)\right) H^{*}(q) \alpha\right\}, \\
V_{\mathrm{Mo}} & =\left\{\alpha \in V \mid \bar{\Gamma}_{\mathrm{Mo}}(\alpha)=\bar{\Gamma}_{\mathrm{Mo}}^{\prime}(\alpha)\right\}, \\
V_{\mathrm{fle}} & =\left\{\alpha \in V \mid H^{*}\left(\Gamma_{\mathrm{fle}}\right)(\tilde{\alpha} \otimes \tilde{\alpha}) \Psi\left(H^{*}\left(\mu_{1}\right)\right)=H^{*}\left(\Gamma_{\mathrm{fle}}^{\prime}\right)(\tilde{\alpha} \otimes \tilde{\alpha}) \Psi\left(H^{*}\left(\mu_{1}\right)\right)\right\},
\end{aligned}
$$

where $m_{2}$ is the product of $H^{*}\left(X_{2} ; \mathbb{Q}\right), \bar{m}_{2}=m_{2}\left(m_{2} \otimes i d\right), \tilde{\alpha}=\Psi^{-1}(\alpha)$ and $\lambda_{2}, \rho_{2}$ are a left inverse and a right inverse of $\operatorname{Hom}_{\mathrm{Alg}}\left(H^{*}\left(X_{2} ; \mathbb{Q}\right), H^{*}\left(X_{2} ; \mathbb{Q}\right)\right)$, respectively. Let

$$
\bar{\Gamma}_{\mathrm{Mo}}(\alpha)=H^{*}\left(\Gamma_{\mathrm{Mo}}(\omega)\right), \bar{\Gamma}_{\mathrm{Mo}}^{\prime}(\alpha)=H^{*}\left(\Gamma_{\mathrm{Mo}}^{\prime}(\omega)\right)
$$

where $\omega: X_{2} \wedge X_{2} \rightarrow X_{1}$ satisfies $H^{*}(\omega)=\alpha$. Then, $V_{\text {inv }}, V_{\text {p.a }}, V_{\text {Mo }}$ and $V_{\text {fle }}$ are subspaces of $V$. 
THEOREM 4.7. The following statements hold.

(1) If $\left(X_{2}, \mu_{2}\right)$ is inversive, then $\operatorname{Im} \overline{H D} \subset V_{\mathrm{inv}}$ and the canonical map $S_{\mathrm{inv}} \rightarrow V_{\mathrm{inv}} / \operatorname{Im} \overline{H D}$ is an isomorphism of algebraic loops.

(2) If $\left(X_{2}, \mu_{2}\right)$ is power associative, then $\operatorname{Im} \overline{H D} \subset V_{\mathrm{p} . \mathrm{a}}$ and the canonical map $S_{\mathrm{p} . \mathrm{a}} \rightarrow$ $V_{\mathrm{p} . \mathrm{a}} / \mathrm{Im} \overline{H D}$ is an isomorphism of algebraic loops.

(3) If $\left(X_{2}, \mu_{2}\right)$ is Moufang, then $\operatorname{Im} \overline{H D} \subset V_{\mathrm{Mo}}$ and the canonical map $S_{\mathrm{Mo}} \rightarrow$ $V_{\mathrm{Mo}} / \mathrm{Im} \overline{H D}$ is an isomorphism of algebraic loops.

(4) If $\left(X_{2}, \mu_{2}\right)$ is flexible, then $\operatorname{Im} \overline{H D} \subset V_{\text {fle }}$ and the canonical map $S_{\text {fle }} \rightarrow V_{\text {fle }} / \operatorname{Im} \overline{H D} i s$ an isomorphism of algebraic loops.

Proof. The assertions follow from Lemma 3.8 and Proposition 4.6.

In the rest of this section, we give some examples of $V, V_{\text {inv }}, V_{\text {p.a }}, V_{\text {Mo }}, V_{\text {fle }}$ and $\operatorname{Im} \overline{H D}$. We suppose that $X_{1}$ is a rational H-space with $H^{*}\left(X_{1} ; \mathbb{Q}\right)=\mathbb{Q}[x]$ or $\Lambda(x)$ in the following two examples.

Example 4.8. Let $X_{2}$ be a rational $\mathrm{H}$-space such that $H^{*}\left(X_{2} ; \mathbb{Q}\right)=\Lambda(y)$. Then we have $V=V_{\text {inv }}=V_{\text {p.a }}=V_{\text {Mo }}=V_{\text {fle }}$ and $\operatorname{Im} \overline{H D}=\{0\}$.

Proof. Since $y^{2}=0$, it follows that $V=V_{\text {inv }}=V_{\text {p.a }}=V_{\mathrm{Mo}}=V_{\text {fle. For }}$ any $\alpha$ in $\operatorname{Hom}_{\mathbb{Q}}(\mathbb{Q}\langle x\rangle, \Lambda(y))$, we may write $\alpha(x)=r y$ for some $r \in \mathbb{Q}$ if $\operatorname{deg} x=\operatorname{deg} y$ and $\alpha(x)=0$ if $\operatorname{deg} x \neq \operatorname{deg} y$ by degree reasons. Then, we have $P_{2}(\alpha(x))=0$; that is, $\operatorname{Im} \overline{H D}=\{0\}$.

Example 4.9. Let $X_{2}$ be a rational H-space such that $H^{*}\left(X_{2} ; \mathbb{Q}\right)=\mathbb{Q}[y]$. Then one has the following.

1. If $\operatorname{deg} x=2 \operatorname{deg} y$, then

$$
V=V_{\text {inv }}=V_{\text {p.a }}=V_{\mathrm{Mo}}=V_{\text {fle }}=\operatorname{Im} \overline{H D} \cong \mathbb{Q} .
$$

2. If $\operatorname{deg} x=3 \operatorname{deg} y$, then

$$
V \cong \mathbb{Q}^{2} \quad \text { and } \quad V_{\text {inv }}=V_{\text {p.a }}=V_{\text {Mo }}=V_{\text {fle }}=\operatorname{Im} \overline{H D} \cong\left\{\left(r_{1}, r_{2}\right) \in \mathbb{Q}^{2} \mid r_{1}=r_{2}\right\} .
$$

3. If $\operatorname{deg} x=m \operatorname{deg} y$ and $m \geq 4$, then $V \cong \mathbb{Q}^{m-1}$,

(i) $\quad V_{\text {inv }} \cong\left\{\begin{array}{l}V(m: \text { even }), \\ \left.\left\{\left(r_{1}, \ldots, r_{m-1}\right) \in \mathbb{Q}^{m-1} \mid \sum_{j=1}^{m-1}(-1)^{j} r_{j}=0\right\} \text { (m: odd }\right),\end{array}\right.$

(ii) $V_{\text {p.a }} \cong\left\{\left(r_{1}, \ldots, r_{m-1}\right) \in \mathbb{Q}^{m-1} \mid \sum_{j=1}^{m-1}\left(2^{j}-2^{m-j}\right) r_{j}=0\right\}$,

(iii) $V_{\mathrm{fle}} \cong\left\{\left(r_{1}, \ldots, r_{m-1}\right) \in \mathbb{Q}^{m-1} \mid r_{l}=r_{m-l}, 1 \leq l \leq m-1\right\}$,

(iv) $\operatorname{Im} \overline{H D} \cong\left\{\left(r_{1}, \ldots, r_{m-1}\right) \in \mathbb{Q}^{m-1} \mid(m-1) ! r_{1}=\cdots=i !(m-i) ! r_{i}=\cdots\right.$ $\left.=(m-1) ! r_{m-1}\right\}$.

4. Other cases, $V=V_{\text {inv }}=V_{\text {p.a }}=V_{\mathrm{Mo}}=V_{\text {fle }}=\operatorname{Im} \overline{H D}=\{0\}$.

Proof. 1. By degree reasons, $V$ is a one-dimensional vector space with the base $\sigma$ defined by $\sigma(x)=y \otimes y$. Suppose that, for any $r \in Q, r \sigma=\overline{H D}(\beta)$ for some $\beta$. We may write $\beta(x)=r_{1} y^{2}$ for some $r_{1} \in \mathbb{Q}$ and then we have $r=2 r_{1}$. This implies that $V=\operatorname{Im} \overline{H D} \cong \mathbb{Q}$. Since $V_{\text {inv }}, V_{\text {p.a }}, V_{\text {Mo }}$ and $V_{\text {fle }}$ include $\operatorname{Im} \overline{H D}$ by Theorem 4.7 , statement 1 is shown. 
2. We choose the basis $\sigma_{1}$ and $\sigma_{2}$ of $\mathrm{V}$ defined by

$$
\sigma_{1}(x)=y \otimes y^{2} \quad \text { and } \quad \sigma_{2}(x)=y^{2} \otimes y .
$$

Then, for any element $\alpha=r_{1} \sigma_{1}+r_{2} \sigma_{2}\left(r_{i} \in \mathbb{Q}\right)$ of $\mathrm{V}$, we have

$$
\begin{aligned}
& m_{2}\left(\lambda_{2} \otimes i d\right) H^{*}(q) \alpha(x)-m_{2}\left(i d \otimes \rho_{2}\right) H^{*}(q) \alpha(x)=\left(-2 r_{1}+2 r_{2}\right) y^{3}, \\
& \bar{m}_{2}\left(H^{*}\left(\mu_{2}\right) \otimes i d\right) H^{*}(q) \alpha(x)-\bar{m}_{2}\left(i d \otimes H^{*}\left(\mu_{2}\right)\right) H^{*}(q) \alpha(x)=\left(-2 r_{1}+2 r_{2}\right) y^{3} .
\end{aligned}
$$

The equalities show that $\alpha \in V_{\text {inv }}\left(\alpha \in V_{\text {p.a }}\right)$ if and only if $r_{1}=r_{2}$. The element $\alpha$ is in $\operatorname{Im} \overline{H D}$ if and only if there exists $\beta$ in $\operatorname{Hom}_{\mathbb{Q}}(\mathbb{Q}\langle x\rangle, \mathbb{Q}\langle y\rangle)$ such that $\alpha=\overline{H D}(\beta)$. Put $\beta(x)=r y^{3}(r \in \mathbb{Q})$, then we have $r_{1}=3 r=r_{2}$. Hence,

$$
V_{\text {p.a }}=V_{\text {inv }}=\operatorname{Im} \overline{H D} \cong\left\{\left(r_{1}, r_{2}\right) \in \mathbb{Q}^{2} \mid r_{1}=r_{2}\right\} .
$$

For this reason, we obtain $V_{\text {inv }}=V_{\text {p.a }}=V_{\text {Mo }}=V_{\text {fle }}=\operatorname{Im} \overline{H D}$ by the implication (2.1) and Theorem 4.7. Therefore statement 2 is shown.

3. We can choose the basis $\sigma_{i}$ of $V$ defined by

$$
\sigma_{i}(x)=y^{i} \otimes y^{m-i} \quad(1 \leq i \leq m-1)
$$

and, for any $\alpha=\sum_{i} r_{i} \sigma_{i}\left(r_{i} \in \mathbb{Q}\right)$ in $V$, we have the following equations:

$$
m_{2}\left(\lambda_{2} \otimes i d\right) H^{*}(q) \alpha(x)-m_{2}\left(i d \otimes \rho_{2}\right) H^{*}(q) \alpha(x)=\sum_{i=1}^{m-1}\left((-1)^{i}-(-1)^{m-i}\right) r_{i} y^{m},
$$

$\bar{m}_{2}\left(H^{*}\left(\mu_{2}\right) \otimes i d\right) H^{*}(q) \alpha(x)-\bar{m}_{2}\left(i d \otimes H^{*}\left(\mu_{2}\right)\right) H^{*}(q) \alpha(x)=\sum_{i=1}^{m-1}\left(2^{i}-2^{m-i}\right) r_{i} y^{m}$.

The equalities show (i) and (ii). A straightforward calculation shows that

$H^{*}\left(\Gamma_{\mathrm{fle}}\right)(\tilde{\alpha} \otimes \tilde{\alpha}) \Psi\left(H^{*}\left(\mu_{1}\right)\right)(x)$

$$
\begin{aligned}
= & H^{*}\left((q \times q)\left(t \times\left(i d_{2} \times \mu_{2} t\right)\left(\Delta_{2} \times i d_{2}\right)\right) \Delta_{2}^{\prime}\right)(\tilde{\alpha}(x) \otimes 1 \otimes 1+1 \otimes 1 \otimes \tilde{\alpha}(x)) \\
= & H^{*}(t)\left(\sum_{i=1}^{m-1} r_{i} y^{i} \otimes y^{m-i}\right)+H^{*}\left(\Delta_{2} \times i d_{2}\right)\left(\sum_{i=1}^{m-1} \sum_{j=0}^{m-i}\left(\begin{array}{c}
m-i \\
j
\end{array}\right) r_{i} y^{i} \otimes y^{m-i-j} \otimes y^{j}\right) \\
= & \sum_{i=1}^{m-1} r_{i} y^{m-i} \otimes y^{i}+\sum_{i=1}^{m-1} \sum_{j=0}^{m-i}\left(\begin{array}{c}
m-i \\
j
\end{array}\right) r_{i} y^{m-j} \otimes y^{j} .
\end{aligned}
$$

We see here that

$$
\begin{aligned}
\sum_{i=1}^{m-1} & \sum_{j=0}^{m-i}\left(\begin{array}{c}
m-i \\
j
\end{array}\right) r_{i} y^{m-j} \otimes y^{j} \\
= & \sum_{j=1}^{m-1}\left(\begin{array}{c}
m-j \\
0
\end{array}\right) r_{j} y^{m} \otimes 1+\sum_{j=1}^{m-1}\left(\begin{array}{c}
m-j \\
1
\end{array}\right) r_{j} y^{m-1} \otimes y+\cdots \\
& +\sum_{j=1}^{m-i}\left(\begin{array}{c}
m-j \\
i
\end{array}\right) r_{j} y^{m-i} \otimes y^{i}+\cdots+r_{1} y \otimes y^{m-1}
\end{aligned}
$$




$$
\begin{aligned}
& =\sum_{j=1}^{m-1} r_{j} y^{m} \otimes 1+\sum_{i=1}^{m-1} \sum_{j=1}^{m-i}\left(\begin{array}{c}
m-j \\
i
\end{array}\right) r_{j} y^{m-i} \otimes y^{i} \\
& =\sum_{j=1}^{m-1} r_{j} y^{m} \otimes 1+\sum_{i=1}^{m-1} \sum_{j=1}^{i}\left(\begin{array}{c}
m-j \\
m-i
\end{array}\right) r_{j} y^{i} \otimes y^{m-i} .
\end{aligned}
$$

Similarly,

$$
\begin{aligned}
H^{*} & \left(\Gamma_{\mathrm{fle}}^{\prime}\right)(\tilde{\alpha} \otimes \tilde{\alpha}) \Psi\left(H^{*}\left(\mu_{1}\right)\right)(x) \\
& =H^{*}\left(\left(i d_{2} \times i d_{2} \times\left(\mu_{2} \times i d_{2}\right)\left(i d_{2} \times t\right)\left(\Delta_{2} \times i d_{2}\right)\right) \Delta_{2}^{\prime}\right)(\tilde{\alpha}(x) \otimes 1 \otimes 1+1 \otimes 1 \otimes \tilde{\alpha}(x)) \\
& =\sum_{i=1}^{m-1} r_{i} y^{i} \otimes y^{m-i}+H^{*}\left(\Delta_{2} \times i d_{2}\right)\left(\sum_{i=1}^{m-1} \sum_{j=0}^{i}\left(\begin{array}{l}
i \\
j
\end{array}\right) y^{j} \otimes y^{m-i} \otimes y^{i-j}\right) \\
& =\sum_{i=1}^{m-1} r_{i} y^{i} \otimes y^{m-i}+\sum_{i=1}^{m-1} \sum_{j=0}^{i}\left(\begin{array}{l}
i \\
j
\end{array}\right) r_{i} y^{m-i+j} \otimes y^{i-j}
\end{aligned}
$$

and

$$
\begin{aligned}
\sum_{i=1}^{m-1} & \sum_{j=0}^{i}\left(\begin{array}{l}
i \\
j
\end{array}\right) r_{i} y^{m-i+j} \otimes y^{i-j} \\
= & \sum_{j=1}^{m-1}\left(\begin{array}{l}
j \\
j
\end{array}\right) r_{j} y^{m} \otimes 1+\sum_{j=1}^{m-1}\left(\begin{array}{c}
j \\
j-1
\end{array}\right) r_{j} y^{m-1} \otimes y+\cdots \\
& +\sum_{j=i}^{m-1}\left(\begin{array}{c}
j \\
j-i
\end{array}\right) r_{j} y^{m-i} \otimes y^{i}+\cdots+r_{m-1} y \otimes y^{m-1} \\
= & \sum_{j=1}^{m-1} r_{j} y^{m} \otimes 1+\sum_{i=1}^{m-1} \sum_{j=i}^{m-1}\left(\begin{array}{c}
j \\
j-i
\end{array}\right) r_{j} y^{m-i} \otimes y^{i} \\
= & \sum_{j=1}^{m-1} r_{j} y^{m} \otimes 1+\sum_{i=1}^{m-1} \sum_{j=m-i}^{m-1}\left(\begin{array}{c}
j \\
j-m+i
\end{array}\right) r_{j} y^{i} \otimes y^{m-i} .
\end{aligned}
$$

Combining these results, we have

$$
\begin{aligned}
H^{*} & \left(\Gamma_{\mathrm{fle}}\right)(\tilde{\alpha} \otimes \tilde{\alpha}) \Psi\left(H^{*}\left(\mu_{1}\right)\right)(x)-H^{*}\left(\Gamma_{\mathrm{fle}}^{\prime}\right)(\tilde{\alpha} \otimes \tilde{\alpha}) \Psi\left(H^{*}\left(\mu_{1}\right)\right)(x) \\
\quad= & \sum_{i=1}^{m-1}\left(r_{m-i}+\sum_{j=1}^{i}\left(\begin{array}{c}
m-j \\
m-i
\end{array}\right) r_{j}-r_{i}-\sum_{j=m-i}^{m-1}\left(\begin{array}{c}
j \\
j-m+i
\end{array}\right) r_{j}\right) y^{i} \otimes y^{m-i} .
\end{aligned}
$$

Thus $\alpha$ is in $V_{\text {fle }}$ if and only if the following equation holds for $i=1,2, \ldots, m-1$ :

$$
r_{m-i}+\sum_{j=1}^{i}\left(\begin{array}{c}
m-j \\
m-i
\end{array}\right) r_{j}=r_{i}+\sum_{j=m-i}^{m-1}\left(\begin{array}{c}
j \\
j-m+i
\end{array}\right) r_{j}
$$

In the case where $i=2$, we have

$$
r_{m-2}+\left(\begin{array}{l}
m-1 \\
m-2
\end{array}\right) r_{1}+\left(\begin{array}{l}
m-2 \\
m-2
\end{array}\right) r_{2}=r_{2}+\left(\begin{array}{c}
m-2 \\
0
\end{array}\right) r_{m-2}+\left(\begin{array}{c}
m-1 \\
1
\end{array}\right) r_{m-1}
$$


by equation (4.1). It follows that $r_{1}=r_{m-1}$. Suppose that the equations $r_{j}=r_{m-j}$ hold for some $1 \leq l \leq m-3$ and any $1 \leq j \leq l$. Then, equation (4.1) in the case where $i=l+2$ shows that

$$
\begin{aligned}
& r_{m-(l+2)}+\sum_{j=1}^{l}\left(\begin{array}{c}
m-j \\
m-(l+2)
\end{array}\right) r_{j}+\left(\begin{array}{l}
m-(l+1) \\
m-(l+2)
\end{array}\right) r_{l+1}+r_{l+2} \\
& =r_{l+2}+\sum_{j=m-l}^{m-1}\left(\begin{array}{c}
j \\
j-m+(l+2)
\end{array}\right) r_{j}+r_{m-(l+2)}+\left(\begin{array}{c}
m-l-1 \\
1
\end{array}\right) r_{m-(l+1)} .
\end{aligned}
$$

The assumption implies that

$$
\sum_{j=1}^{l}\left(\begin{array}{c}
m-j \\
m-(l+2)
\end{array}\right) r_{j}=\sum_{j=1}^{l}\left(\begin{array}{c}
m-j \\
m-(l+2)
\end{array}\right) r_{m-j}=\sum_{j=m-l}^{m-1}\left(\begin{array}{c}
j \\
j-m+(l+2)
\end{array}\right) r_{j}
$$

and it follows that $r_{l+1}=r_{m-(l+1)}$. Conversely, we see that the equalities $r_{l}=r_{m-l}$ for $1 \leq l \leq m-1$ imply the equations

$$
\sum_{j=1}^{i}\left(\begin{array}{c}
m-j \\
m-i
\end{array}\right) r_{j}=\sum_{j=1}^{i}\left(\begin{array}{c}
m-j \\
m-i
\end{array}\right) r_{m-j}=\sum_{j=m-i}^{m-1}\left(\begin{array}{c}
j \\
j-m+i
\end{array}\right) r_{j}
$$

that is, equation (4.1) holds. Hence (iii) is shown. If $\alpha$ is in $\operatorname{Im} \overline{H D}$, that is, $\alpha=\overline{H D}(\beta)$ for some $\beta$ in $\operatorname{Hom}_{\mathbb{Q}}(\mathbb{Q}\langle x\rangle, \mathbb{Q}\langle y\rangle)$, then we may write $\beta(x)=r y^{m}$ for some $r \in \mathbb{Q}$ and the equation

$$
\alpha(x)=\overline{H D}(\beta)(x)=\sum_{j=1}^{m-1}\left(\begin{array}{c}
m \\
j
\end{array}\right) r y^{j} \otimes y^{m-j}
$$

shows that $r_{i}=\left(\begin{array}{c}m \\ i\end{array}\right) r$ for any $i$. Therefore (iv) is shown.

4. We readily see that $V=\{0\}$ by degree reasons. It implies the statement 4 .

Proof of Assertion 1.1. If $n=k m$ and $k \geq 4$ is even, by Example 4.9, we have

$$
V_{\mathrm{Mo}} / \operatorname{Im} H D \subseteq V_{\mathrm{fle}} / \operatorname{Im} H D \subsetneq V_{\text {p.a }} / \operatorname{Im} H D \subsetneq V / \operatorname{Im} H D=V_{\text {inv }} / \operatorname{Im} H D .
$$

Thus statement (1) holds. A similar argument shows the other cases.

Proof of Corollary 1.2. The map

$$
l: C H\left(K(\mathbb{Z}, 2 n) ; S^{n}\right) \longrightarrow C H\left(K(\mathbb{Q}, 2 n) ; S_{\mathbb{Q}}^{n}\right)
$$

induced by the localization maps $K(\mathbb{Z}, 2 n) \rightarrow K(\mathbb{Q}, 2 n)$ and $S^{n} \rightarrow S_{\mathbb{Q}}^{n}$ is injective since $C H\left(K(\mathbb{Z}, 2 n) ; S^{n}\right) \cong \mathbb{Z}$ and $C H\left(K(\mathbb{Q}, 2 n) ; S^{n}\right) \cong \mathbb{Q}$. We see that the map $l$ is indeed the inclusion $\mathbb{Z} \hookrightarrow \mathbb{Q}$. On the other hand, by Example 4.8, any central H-extensions of $K(\mathbb{Q}, 2 n)$ by $S_{\mathbb{Q}}^{n}$ are Moufang. Therefore, any central H-extensions of $K(\mathbb{Z} ; 2 n)$ by $S^{n}$ are also Moufang. Since $C H\left(K(\mathbb{Z}, 2 n) ; S^{n}\right)$ is a subset of the set of essentially different multiplications of $K(\mathbb{Z}, 2 n) \times S^{n}$, this completes the proof. 
Acknowledgements. The present paper is the author's Master's thesis written at Shinshu University. The author would like to thank his adviser, Katsuhiko Kuribayashi, for his encouragement and support. The author is also grateful to Ryo Takahashi for his helpful comments, and to the referees for their careful reading and valuable remarks.

\title{
REFERENCES
}

[1] M. Arkowitz and G. Lupton. Loop-theoretic properties of H-spaces. Math. Proc. Camb. Phil. Soc. 110 (1991), 121-136.

[2] R. H. Bruck. Contributions to the theory of loops. Trans. Amer. Math. Soc. 60 (1946), 245-354.

[3] C. R. Curjel. On the H-space structures of finite complexes. Comm. Math. Helv. 24 (1968), 1-17.

[4] I. M. James. On H-spaces and their homotopy groups. Quart. J. Math. 11 (1960), 161-179.

[5] H. Kachi. Central extensions of H-spaces. Kyushu J. Math. 50 (1996), 249-262.

[6] J. W. Milnor and J. C. Moore. On the structure of Hopf algebra. Ann. of Math. (2) 81 (1965), 211-264.

[7] C. W. Norman. Homotopy loops. Topology 2 (1963), 23-43.

[8] H. Scheerer. On rationalized $\mathrm{H}-$ and co-H-spaces, with an appendix on decomposable $\mathrm{H}-$ and co-H-spaces. Manuscripta Math. 51 (1984), 63-87.

[9] J. D. H. Smith. An Introduction to Quasigroups and their Representations (Studies in Advanced Mathematics). Chapman \& Hall/CRC, Boca Raton, FL, 2007.

[10] E. H. Spanier. Algebraic Topology. McGraw-Hill, 1966.

[11] A. Zabrodsky. Hopf Spaces (Math. Studies, 22). North-Holland, Amsterdam, 1976.

\author{
Takahito Naito \\ Interdisciplinary Graduate School of Science and Technology \\ Shinshu University \\ 3-1-1 Asahi \\ Matsumoto \\ Nagano 390-8621 \\ Japan \\ (E-mail:naito@math.shinshu-u.ac.jp)
}

